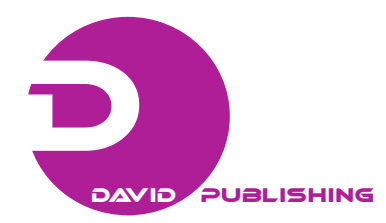

\title{
Synthesis of Zirconia Nanoparticles in Distilled Water Solution by Laser Ablation Technique
}

\author{
Adel K. Mahmoud ${ }^{1}$, Zainab Fadhill², Suha Ibrahim Al-nassar ${ }^{2}$, Furat Ibrahim Husein ${ }^{3}$, Erhan Akman $^{4}$ and Arif \\ Demir $^{5}$ \\ 1. Mechanical Engineering Department, College of Engineering, Diyala University, Diyala, Iraq \\ 2. Laser Institute for Postgraduate Studies, Baghdad University, Baghdad, Iraq \\ 3. Al-Khwarizmi College of Engineering, Baghdad University, Baghdad, Iraq \\ 4. Laser Technologies Research and Application Center, Kocaeli University, Kocaeli 41275, Turkey \\ 5. Electro-Optics Systems Engineering, Kocaeli University, Kocaeli, Umuttepe 41380, Turkey
}

Received: March 09, 2013 / Accepted: April 13, 2013 / Published: June 25, 2013.

\begin{abstract}
Pulsed laser ablation in liquid (PLAL) has become an increasingly important technique for metals production and metal oxides nanoparticles (NPs) and others. This technique has its many advantages compared with other conventional techniques (physical and chemical). This work was devoted for production of zirconia $\left(\mathrm{ZrO}_{2}\right)$ nanoparticles via PLAL technique from a solid zirconium target immersed in a wet environment in order to study the effect of this environment on the optical properties and structure of $\mathrm{ZrO}_{2}$ nanoparticles. The solutions which used for this purpose is distilled water (D.W). The produces NPs were characterized by mean of many tests such as UV-visible (UV-Vis.), transmission electron microscope (TEM) and Z-Potential. The UV-Vis. A spectrum has atwavelength is $271 \mathrm{~nm}$. The TEM test shows less than $10 \mathrm{~nm}$ average particle sizes with spherical and irregular shapes. Z-Potential test shows value about $+56.1 \mathrm{mV}$ which indicate for NPs stability with extremely low agglomeration solution.
\end{abstract}

Key words: Zirconia nanoparticles, distilled water solution, pulsed laser ablation technique.

\section{Introduction}

The properties and behavior of materials at the nano-scale or level vary greatly when compared to micro levels. The properties of nanoparticles show great differences in electrical, optical, magnetic and chemical properties from the bulk material of which they are made. Accordingly nanocrystalline zirconia is fundamentally different from those of conventional micrometer-sized zirconia because of the nanosize effect and high surface-to-volume ratio [1]. They exhibit increased strength and ductility, enhanced diffusivity and increased edges and corners, all beneficial to applications in sensors, electronics, medical, etc. [2].

Correspondingauthor: Adel K. Mahmoud, Ph.D., associate professor, research fields: laser processing and surface engineering. E-mail: dr.eng.adel@uodiyala.edu.iq.
Pulsed laser ablation in liquid represents one of the most important, effective and simple technique for preparing metal, metal oxide nanoparticles. It has many advantages compared with other conventional physical andchemical methods like purity, stability of the fabricated nanoparticle colloids, simple chemical preparation and do not require a vacuum chamber.

It is the most flexible and promising technique because of its ability to control nanoparticles (NPs) size by optimizing the operating conditions such as irradiation time, pulse duration, energy density, wavelength, etc. [3]. Generating nanoparticles through pulsed laser ablation in liquid (PLAL) technique passes through threefundamental steps. Firstly, plasma generates due to extreme heating during the interaction of laser with the matter. Secondly, the ultrasonic adiabatic plasma expand leads to quick 
cooling of the plume region and hence to the formation of nanoparticles clusters. Finally, after plasma extinguishing the formed nanoparticles clusters encounter and interact with the solvent and surfactant molecules in the surrounding solution.

The concept of producing metal oxides using PLAL technique was demonstrated in 1987, accordingly iron and tantalum oxides were formed on (or from) target surfaces immersed in water using a third harmonic Q-switched ruby pulsed laser [4]. zirconia is one of the metal oxides nanoparticles; these particles have many characteristics such as their wide band gap 5.0-5.5 eV transition metal oxide, excellent mechanical, thermal, optical and electrical properties. Pure crystalline zirconia exhibits three crystallographic phases: monoclinic $\left(\mathrm{m}-\mathrm{ZrO}_{2}\right)$, tetragonal (t-ZrO $\mathrm{Zr}_{2}$ ) and cubic $\left(\mathrm{c}-\mathrm{ZrO}_{2}\right)$ phases [5].

This work was focused on the effect of distilled water in controlling the size and stability of generated NPs as well as agglomerations reduction. Distilled water (D.W) was used as an ablation environment to capture and store the synthesized NPs. Distilled water represents the base medium for many chemical reactions occur during nanoparticles formation.

\section{Experiments}

Fig. 1 shows the schematic diagram of the PLAL experiment setup for the synthesis colloidal solution of zirconia NPs. The laser used in this work is a nanosecond Q-Switched Nd:YAG laser type Surelite Continuum Laser at Kocaeli University Laser Technologies Research and Application Center (LATARUM). It operates at 1,064 nm wavelength, 10 $\mathrm{Hz}$ pulse repetition rate and 6 ns pulse duration at 1 $\mathrm{J} /$ pulse as a maximum output. This laser beam is focused via a $100 \mathrm{~mm}$ focal length focusing lens to a minimum spot size at a solid zirconium target (purity 99.99\%). The zirconium target was fixed by a fixture inside the flask and immersed at $10 \mathrm{~mm}$ depth in the solution inside the flask. A well designed and fabricated rotator mechanism used to rotate the flask in order to maintain the continuous changing of the focused laser spot position at the target. A magnetic stirrer rotator was placed in the solution rotates at 600 r.p.m. to ensure uniform irradiation on target and the movement of water that can enhance ablated particle diffusion also to disperse the produced NPs. Laser power was measured via a power meter type Newport 841-PE, the measurement was obtained at two locations very near to the final stage of the laser apparatus and before the focusing lens to evaluate the losses of the power in the beam delivery unit. Before starting the experiment the zirconium target was cleaned by ultrasonic cleaning device type EMAG 50 HC then wiped with acetone and ethanol solvents. D.W solution was used as the wet environment to study its effects on the produced NPs. The experiments of producing zirconia NP were done at 20 min exposure time, $120 \mathrm{~mJ}$ pulse energy, 6 ns pulse duration and $10 \mathrm{~Hz}$ repetition rate. A number of tests were done to characterize the produced zirconia NPs, before doing the tests the sample was placed in the ultrasonic cleaner to ensure the homogeneity of the NPs solution. UV-visible extinction spectrum of the colloidal solutions was recorded using a spectrophotometer type Varian Cary-50 UV-Visible. NPs size, morphology and distribution were examined by Transmission Electron Microscope imaging device. Malvern Nano ZS90 analytical technique test was done for potential measurements and JEOL JSM 6400F for electron microscopy.

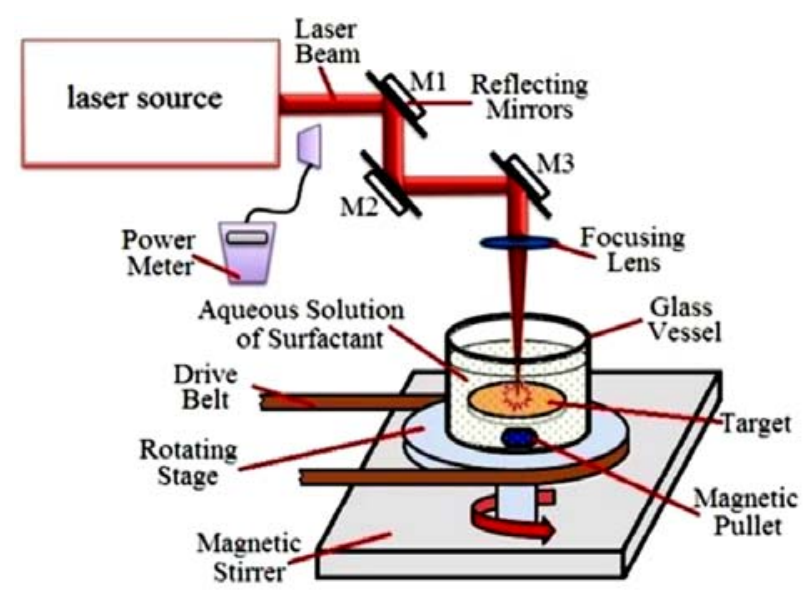

Fig. 1 Mapping experimental set up of nanosecond laser ablation method. 


\section{Results and Discussion}

In the experiments of this research work, laserablation of the zirconium target in D.W solution was investigated this process accompanied by the production of a plasma plume, visible to the eye, near the target surface. Therefore after laser irradiation time 20 min, a milky colloidal solution of oxide-based nanomaterials was obtained. Change of color of colloidal solution of $\mathrm{ZrO}_{2}$ to a milky color indicates that nanosized colloidal particles have produced. The shape and the size distributions of $\mathrm{ZrO}_{2}$ nanoparticles in D.Wsolution were characterized by many inspections such as (TEM, UV-visible and Z-Potential).

Fig. 2 displays the absorption spectra of zirconia produced in D.W media ( $\lambda=1,064 \mathrm{~nm}, E=120$ $\mathrm{mJ} /$ pulse, $t=20 \mathrm{~min}$ ). UV-visible absorption spectrum asa synthesized colloidal solution of nanoparticles has a peak wavelength $271 \mathrm{~nm}$. The UV peak at $271 \mathrm{~nm}$ is due to the transition of an inner shell electron to the conduction, which is almost reported in the case of UV-visible absorption of all metal and metal oxide nanoparticles [6].

The solution of nanoparticle which produced in D.W showed fine bubbles in front of target after first 10 min of ablation. These bobbles apparently prohibited the laser energy to be absorbed by the target, which could easily be realized by extremely diminished noises of impacts. Also it seemed to be stable, and little pale yellowish sediment could be observed at the bottom of its glassy container after 4-7 days. zirconia is a direct band gap insulator with two bands to band transitions and the absorption behavior could arise from the surface trap state or defects [7] and by our calculation the band gap energy for cubic zirconia we obtained the value $(4.375 \mathrm{eV}$ in photon energy) at absorption spectra (271 nm). These obtained data agree with the information that the band gap energy for cubic zirconia is 3.8-6.1 eV [2].

Many researches showed that oxygen vacancies play an important role in the stabilization of nanocrystalline cubic and tetragonal zirconia at room temperature [2]. As the results above, nanosecond PLAL is favorable for the production and stabilization of metastable zirconia. Moreover, the particles existed in nanometer scale and there should be more oxygen vacancies in these nanocrystallites due to the large surface-to-volume ratio. The absorption behavior of the direct band gap insulator zirconia as shown in Fig. 2 confirmed the presence of oxygen defects [2]. According to the Fig. 2 the optical absorption of colloidal $\mathrm{ZrO}_{2}$ in D.W solution reveals a broad band with a long tail toward the $271 \mathrm{~nm}$ wavelength, indicating the formation of inhomogeneous sizes and a particle coagulation shift in wavelength of maximum optical extinction and they exhibited a weak peak in infrared region that originates from elongation and agglomeration of nanoparticles [8].

The formation of approximately spherical $\mathrm{ZrO}_{2}$ nanoparticles during pulsed laser ablation technique in D.W solution was confirmed through TEM images. Fig. 3 shows a TEM image and size distribution of $\mathrm{ZrO}_{2}$ nanoparticles were a mixture of different sizes and shapes in D.W solution that the particles are almost spherical or irregular spherical with large number of smaller particle size less than $10 \mathrm{~nm}$. In this research work, the calculated average size diameters of $\mathrm{ZrO}_{2}$ NPs are about $7.61 \mathrm{~nm}$ in D.W as shown in Fig. 3.

NPs are formed during the process of PLAL by many steps staring in phase transition, nucleation and

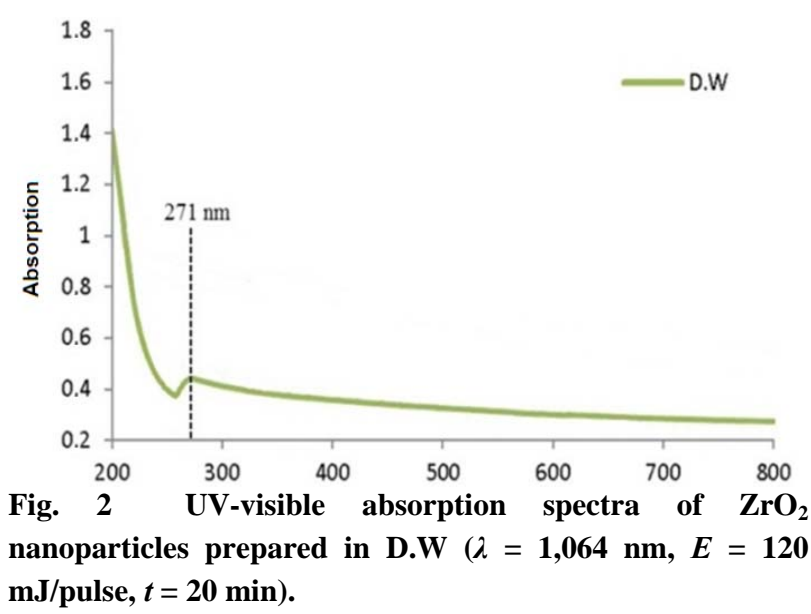



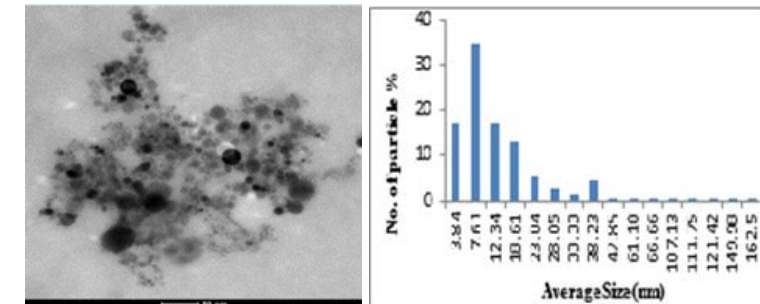

Fig. 3 TEM and size distribution $\mathrm{ZrO}_{2}$ nanoparticles produced using nanosecond laser beam $(\lambda=1,064 \mathbf{~ n m}, E=$ $120 \mathrm{~mJ} /$ pulse, $t=20 \mathrm{~min}$ ) in D.W.

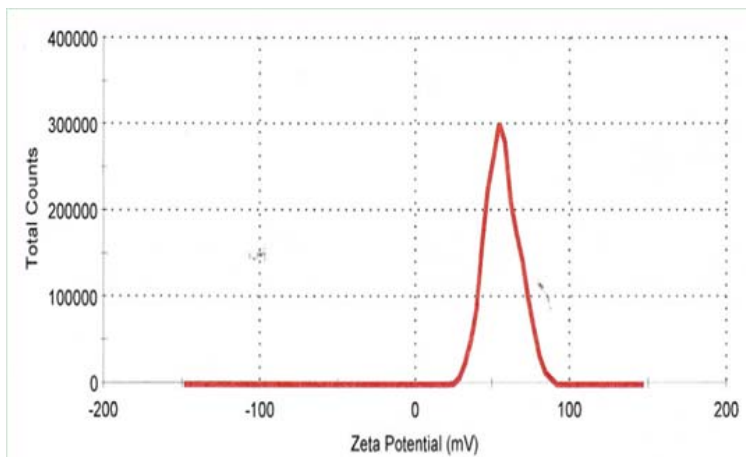

Fig. 4 Zeta potential distributions of $\mathrm{ZrO}_{2}$ nanoparticles produced by using nanosecond laser beam $(\lambda=1,064 \mathrm{~nm}, E$ $=120 \mathrm{~mJ} /$ pulse, $t=20 \mathrm{~min}$ ) in $\mathrm{D} . \mathrm{W}$ solution.

crystal growth of emitted materials from the target plate. These produced NPs formed were adsorbed by the solution and this adsorption can prevent aggregation and growth of the particles [8,9]. When the metal is reactive with the surrounding liquid, such as water, more complicated chemical reactions between the ablated species and the molecules of water are involved to form oxides or hydroxides [10]. In this process, it was expected that the ablated $\mathrm{Zr}$ in the form of ions, atoms or clusters would strongly react with the water molecules to form $\mathrm{ZrO}_{2}$ molecules, which rapidly were quenched in the liquid solution to form NPs.

Measurements of zeta potential were also carried out in order to study the stability of physical property which is given the net surface charge of the nanoparticles, when these particles inside the solution repelling each other's since produced a coulomb explosion between the charges of the nanoparticles giving rise to no tendency for the particles to agglomerate. The criteria of stability of NPs are measured when the values of zeta potential ranged from higher than $+30 \mathrm{mV}$ to lower than $-30 \mathrm{mV}$ [20] Fig. 4 shows zeta potential distributions of $\mathrm{ZrO}_{2}$ nanoparticles illuminated by $1,064 \mathrm{~nm}$ in D.W solution, the value of zeta potential of D.W solution is $56.1 \mathrm{mV}$, this result indicating thenanoparticles produced D.W are stable with extremely low agglomeration nanoparticles solution.

\section{Conclusions}

In summary, this research work has successfully produced pure zirconia nanocrystallites by using a simple method of nanosecond pulsed laser ablation in D.W. solution with a large number of smaller sizes of NPs less than $10 \mathrm{~nm}$. The energy gap of produced $\mathrm{ZrO}_{2}$ NPs in D.W is $4.375 \mathrm{eV}$ photon energy at absorption spectra $(271 \mathrm{~nm})$. These obtained data agree with the information that the band gap energy for cubic zirconia. The value of zeta potential analysis in D.W solution is $56.1 \mathrm{mV}$, with extremely low agglomeration nanoparticles solution and stable. This convenient synthesis strategy can be applied as a general approach that $\mathrm{ZrO}_{2}$ NPs have attracted significant interest of materials scientists and physicists due to their special properties and have attained a great importance in several technological applications such medical and biological applications.

\section{Acknowledgments}

This work was supported by Kocaeli University Laser Technologies Research and Application Center (LATARUM) in Turkey and the Ministry of Higher Education of Iraq.

\section{References}

[1] V. Piriyawong, V. Thongpool, P. Asanithi, P. Limsuwan, Effect of laser pulse energy on the formation of alumina nanoparticles synthesized by laser ablation inwater, Surface Science Direct 32 (2012) 1107-1112.

[2] R.K. Swarnkar, S.C. Singh, R. Gopal, Optical characterizations of copper oxide nanomaterial, in: International Conference on Optics and Photonics, Chandigarh, India, 2009, pp. 1-3.

[3] Z. Liu1, Y. Yuan, S. Khan, A.Abdolvand, D. Whitehead, M. Schmidt, et al., Generation of metal-oxide 
nanoparticles using continuous-wave fiber laser Ablation in liquid, Journal Micromech Microeng. 19 (2009) 1-7.

[4] F. Mafune, J. Kohno, Y. Take da, T. Kondow, Structure and stability of silver nanoparticles in aqueous solution produced by laser ablation, Journal Physics Chemistry B 104 (35) (2000) 8333-8337.

[5] T. Tsuji, D.H. Thang, Y. Okazaki, M. Nakanishi, Y. Tsuboi, M. Tsuji, Preparation of silver nanoparticles by laser ablation in polyvinylpyrrolidone solutions, Applied Surface Science 254 (2008) 5224-5230.

[6] S.C. Singh, R.K.Swarnkar, R.Gopal, Zn/ZnO core/shell nanoparticles synthesized by laser ablation in aqueous environment: Optical and structural characterizations, Indian Academy of Sciences 331 (2010) 21-26.

[7] D. Tan, G. Lin, Y. Liu, Y. Teng, Synthesis of nanocrystalline cubic zirconia using femtosecond laser ablation, Journal Nanopart. Research 13 (2011) 1183-1190.

[8] S.Z. Khan, Y.D.Yuan, A.Abdolvand, M. Schmidt, P. Crouse, L. Li, et al., Generation and characterization of NiOnanoparticles by continuous wave fiber laser ablation in liquid, Journal Nanopart.Res. 11 (2008) 1421-1427.

[9] Q.A. Drmosh, M.A. Gondal, Z.H. Yamani, T.A. Saleh, Spectroscopic characterization approach to study surfactants effect on $\mathrm{ZnO}_{2}$ nanoparticles synthesis by laser ablation process, Applied Surface Science 256 (2010) 4661-4666.

[10] E. Akman, B. GencOztoprak, M. Gunes, E. Kacar, A. Demir, Effect of femtosecond Ti: Sapphire laser wavelengths on plasmonic behavior and size evolution of silver nanoparticles, Photon Nanostruct: Fundam Appl. 9 (2011) 276-286. 